Supplement of Nat. Hazards Earth Syst. Sci., 14, 1553-1564, 2014

http://www.nat-hazards-earth-syst-sci.net/14/1553/2014/

doi:10.5194/nhess-14-1553-2014-supplement

(C) Author(s) 2014. CC Attribution 3.0 License.

(c) (1)
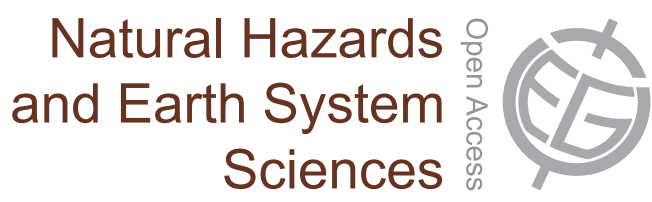

Supplement of

\title{
An assessment of landslide susceptibility in the Faifa area, Saudi Arabia, using remote sensing and GIS techniques
}

\section{T. Alharbi et al.}

Correspondence to: M. Sultan (mohamed.sultan@wmich.edu) 
Table S1. Identification of slope dip directions that are controlled by fracture planes dipping in the same direction.

\begin{tabular}{|c|c|c|c|c|c|c|c|c|c|c|}
\hline 1 & 2 & 3 & 4 & 5 & 6 & 7 & 8 & 9 & 10 & 11 \\
\hline 1.0 & 69 & 352 & 4 & 1 & 100.0 & & & & & \\
\hline 3.0 & 70 & 344 & 14 & 4 & 50.0 & 1 & 1 & 2 & 70 & 348 \\
\hline 10.0 & 87 & 2 & 14 & 3 & 40.0 & \multirow{3}{*}{2} & \multirow{3}{*}{2} & 10 & 87 & 2 \\
\hline 11.0 & 53 & 353 & 4 & 1 & 100.0 & & & \multirow[t]{2}{*}{12} & \multirow[t]{2}{*}{49} & \multirow[t]{2}{*}{3} \\
\hline 12.0 & 46 & 12 & 10 & 3 & 100.0 & & & & & \\
\hline 20.0 & 53 & 27 & 13 & 5 & 60.0 & \multirow{5}{*}{3} & \multirow{5}{*}{2} & \multirow{4}{*}{23} & \multirow{4}{*}{53} & \multirow{4}{*}{32} \\
\hline 21.0 & 46 & 37 & 4 & 1 & 100.0 & & & & & \\
\hline 24.0 & 58 & 33 & 3 & 1 & 100.0 & & & & & \\
\hline 25.0 & 53 & 31 & 12 & 4 & 75.0 & & & & & \\
\hline 26.0 & 20 & 14 & 3 & 1 & 100.0 & & & 26 & 20 & 14 \\
\hline 48.0 & 48 & 57 & 6 & 2 & 50.0 & \multirow{4}{*}{4} & \multirow{4}{*}{3} & & & \\
\hline 49.0 & 37 & 44 & 4 & 1 & 100.0 & & & 49 & 43 & 51 \\
\hline 51.0 & 88 & 52 & 6 & 2 & 50.0 & & & 51 & 88 & 52 \\
\hline 52.0 & 10 & 49 & 3 & 1 & 100.0 & & & 52 & 10 & 49 \\
\hline 55.0 & 66 & 57 & 14 & 4 & 75.0 & \multirow[b]{2}{*}{5} & \multirow[b]{2}{*}{1} & \multirow[b]{2}{*}{58} & \multirow[b]{2}{*}{61} & \multirow[b]{2}{*}{57} \\
\hline 60.0 & 56 & 57 & 6 & 2 & 100.0 & & & & & \\
\hline 64.0 & 87 & 65 & 4 & 1 & 100.0 & \multirow{3}{*}{6} & \multirow{3}{*}{1} & \multirow{3}{*}{65} & \multirow{3}{*}{82} & \multirow{3}{*}{71} \\
\hline 65.0 & 83 & 76 & 6 & 2 & 50.0 & & & & & \\
\hline 67.0 & 77 & 72 & 11 & 3 & 66.7 & & & & & \\
\hline 70.0 & 58 & 74 & 10 & 3 & 66.7 & \multirow{3}{*}{7} & \multirow{3}{*}{1} & \multirow{3}{*}{72} & \multirow{3}{*}{57} & \multirow{3}{*}{68} \\
\hline 72.0 & 57 & 68 & 11 & 3 & 100.0 & & & & & \\
\hline 74.0 & 56 & 62 & 9 & 2 & 50.0 & & & & & \\
\hline \multirow[t]{2}{*}{77.0} & \multirow[t]{2}{*}{41} & \multirow[t]{2}{*}{74} & 4 & 1 & 100.0 & \multirow[b]{3}{*}{8} & & & & \\
\hline & & & 4 & 1 & 100.0 & & & 77 & 41 & 74 \\
\hline 84.0 & 68 & 95 & 7 & 2 & 100.0 & & 2 & & & \\
\hline
\end{tabular}




\begin{tabular}{|c|c|c|c|c|c|c|c|c|c|c|}
\hline 85.0 & 53 & 89 & 10 & 3 & 100.0 & & & 85 & 61 & 92 \\
\hline 95.0 & 54 & 96 & 6 & 2 & 66.7 & \multirow{7}{*}{9} & \multirow{7}{*}{2} & \multirow[b]{3}{*}{100} & \multirow[b]{3}{*}{57} & \multirow[b]{3}{*}{99} \\
\hline 101.0 & 58 & 96 & 5 & 1 & 100.0 & & & & & \\
\hline 105.0 & 60 & 105 & 3 & 1 & 100.0 & & & & & \\
\hline 97.0 & 32 & 97 & 4 & 1 & 100.0 & & & \multirow[b]{4}{*}{101} & \multirow[b]{4}{*}{39} & \multirow[b]{4}{*}{100} \\
\hline 99.0 & 44 & 83 & 4 & 1 & 100.0 & & & & & \\
\hline 100.0 & 43 & 109 & 15 & 5 & 100.0 & & & & & \\
\hline 107.0 & 38 & 110 & 3 & 1 & 100.0 & & & & & \\
\hline 115.0 & 34 & 130 & 6 & 2 & 50.0 & \multirow[t]{2}{*}{10} & \multirow[t]{2}{*}{2} & 115 & 10 & 2 \\
\hline 114.0 & 60 & 120 & 11 & 3 & 100.0 & & & 114 & 60 & 120 \\
\hline 135.0 & 44 & 144 & 16 & 5 & 85.7 & \multirow{7}{*}{11} & \multirow{7}{*}{3} & \multirow[b]{4}{*}{142} & \multirow[b]{4}{*}{48} & \multirow[b]{4}{*}{144} \\
\hline 145.0 & 53 & 139 & 13 & 5 & 80.0 & & & & & \\
\hline \multirow[t]{2}{*}{146.0} & 46 & 150 & 3 & 1 & 100.0 & & & & & \\
\hline & & & 3 & 1 & 100.0 & & & & & \\
\hline 138.0 & 24 & 135 & 3 & 1 & 100.0 & & & & & \\
\hline 140.0 & 30 & 140 & 7 & 2 & 50.0 & & & 139 & 27 & 138 \\
\hline 136.0 & 86 & 134 & 3 & 1 & 100.0 & & & 136 & 86 & 134 \\
\hline 150.0 & 43 & 156 & 3 & 1 & 100.0 & \multirow{13}{*}{12} & \multirow{13}{*}{3} & \multirow[b]{9}{*}{170} & \multirow[b]{5}{*}{39} & \multirow[b]{5}{*}{158} \\
\hline 157.0 & 38 & 161 & 5 & 1 & 100.0 & & & & & \\
\hline 160.0 & 46 & 150 & 4 & 1 & 100.0 & & & & & \\
\hline 165.0 & 43 & 155 & 8 & 3 & 66.7 & & & & & \\
\hline 181.0 & 27 & 166 & 3 & 1 & 100.0 & & & & & \\
\hline 158.0 & 68 & 158 & 3 & 1 & 100.0 & & & & & \\
\hline 163.0 & 63 & 151 & 10 & 3 & 66.7 & & & & & \\
\hline 184.0 & 49 & 199 & 8 & 2 & 50.0 & & & & & \\
\hline 176.0 & 67 & 167 & 7 & 2 & 100.0 & & & & 62 & 169 \\
\hline 170.0 & 70 & 168 & 6 & 2 & 100.0 & & & & & \\
\hline 180.0 & 78 & 175 & 4 & 1 & 100.0 & & & & & \\
\hline 188.0 & 81 & 195 & 4 & 1 & 100.0 & & & & & \\
\hline 190.0 & 72 & 196 & 2 & 1 & 100.0 & & & & & \\
\hline
\end{tabular}




\begin{tabular}{|c|c|c|c|c|c|c|c|c|c|c|}
\hline 155.0 & 86 & 135 & 5 & 2 & 50.0 & & & 177 & 77 & 174 \\
\hline 198.0 & 30 & 180 & 4 & 1 & 100.0 & \multirow{5}{*}{13} & \multirow{5}{*}{3} & 198 & 30 & 180 \\
\hline 200.0 & 37 & 218 & 4 & 1 & 100.0 & & & & & \\
\hline 201.0 & 58 & 185 & 3 & 1 & 100.0 & & & & & \\
\hline 205.0 & 52 & 207 & 14 & 4 & 100.0 & & & 202 & 49 & 203 \\
\hline 197.0 & 81 & 204 & 3 & 1 & 100.0 & & & 197 & 81 & 204 \\
\hline 212.0 & 65 & 200 & 6 & 2 & 100.0 & \multirow{4}{*}{14} & \multirow{4}{*}{2} & & & \\
\hline 213.0 & 60 & 210 & 12 & 4 & 75.0 & & & & & \\
\hline 215.0 & 67 & 204 & 6 & 2 & 50.0 & & & 213 & 64 & 204 \\
\hline 216.0 & 87 & 209 & 4 & 1 & 100.0 & & & 216 & 87 & 209 \\
\hline 219.0 & 57 & 217 & 9 & 2 & 100.0 & & & & & \\
\hline 220.0 & 69 & 186 & 15 & 4 & 100.0 & 15 & 1 & 220 & 63 & 201 \\
\hline 237.0 & 72 & 228 & 7 & 2 & 50.0 & \multirow{7}{*}{16} & \multirow{7}{*}{3} & 237 & 72 & 228 \\
\hline 235.0 & 88 & 237 & 13 & 4 & 80.0 & & & & & \\
\hline 238.0 & 60 & 246 & 8 & 2 & 100.0 & & & & & \\
\hline 240.0 & 61 & 245 & 9 & 3 & 100.0 & & & & & \\
\hline 242.0 & 72 & 242 & 3 & 1 & 100.0 & & & & & \\
\hline 243.0 & 62 & 243 & 3 & 1 & 100.0 & & & 240 & 69 & 242 \\
\hline 236.0 & 84 & 223 & 4 & 1 & 100.0 & & & 236 & 84 & 223 \\
\hline 245.0 & 64 & 235 & 4 & 1 & 100.0 & \multirow[t]{2}{*}{17} & \multirow[t]{2}{*}{2} & 245.0 & 64 & 235 \\
\hline 247.0 & 74 & 234 & 6 & 2 & 100.0 & & & 247.0 & 74 & 234 \\
\hline 250.0 & 63 & 244 & 11 & 4 & 75.0 & \multirow[b]{2}{*}{18} & \multirow[b]{2}{*}{1} & \multirow[b]{2}{*}{251} & \multirow[b]{2}{*}{65} & \multirow[b]{2}{*}{253} \\
\hline 252.0 & 68 & 262 & 7 & 2 & 100.0 & & & & & \\
\hline 264.0 & 54 & 284 & 8 & 2 & 100.0 & \multirow[b]{3}{*}{19} & \multirow[b]{3}{*}{1} & \multirow[b]{3}{*}{265} & \multirow[b]{3}{*}{53} & \multirow[b]{3}{*}{269} \\
\hline 265.0 & 51 & 261 & 9 & 3 & 66.7 & & & & & \\
\hline 266.0 & 55 & 263 & 9 & 2 & 100.0 & & & & & \\
\hline 275.0 & 72 & 274 & 3 & 1 & 100.0 & \multirow{3}{*}{20} & \multirow{3}{*}{2} & & & \\
\hline 276.0 & 65 & 283 & 4 & 1 & 100.0 & & & 276 & 69 & 279 \\
\hline 277.0 & 15 & 288 & 3 & 1 & 100.0 & & & 277.0 & 15 & 288 \\
\hline
\end{tabular}




\begin{tabular}{|c|c|c|c|c|c|c|c|c|c|c|}
\hline 286.0 & 47 & 306 & 5 & 1 & 100.0 & 21 & 1 & 287 & 46 & 307 \\
\hline 287.0 & 44 & 307 & 3 & 1 & 100.0 & & & & & \\
\hline 311.0 & 55 & 307 & 8 & 2 & 50.0 & \multirow{8}{*}{22} & \multirow{8}{*}{3} & & & \\
\hline 312.0 & 45 & 312 & 6 & 2 & 100.0 & & & & & \\
\hline 313.0 & 52 & 311 & 3 & 1 & 100.0 & & & & & \\
\hline 315.0 & 56 & 325 & 6 & 2 & 100.0 & & & & & \\
\hline 319.0 & 40 & 307 & 3 & 1 & 100.0 & & & & & \\
\hline 322.0 & 48 & 330 & 6 & 2 & 50.0 & & & 315.3 & 49 & 315 \\
\hline 318.0 & 78 & 318 & 6 & 2 & 100.0 & & & 318 & 78 & 318 \\
\hline 320.0 & 10 & 313 & 13 & 4 & 50.0 & & & 320.0 & 10 & 313 \\
\hline 328.0 & 38 & 323 & 3 & 1 & 100.0 & \multirow{4}{*}{23} & \multirow{4}{*}{3} & 328 & 38 & 323 \\
\hline 325.0 & 53 & 333 & 23 & 7 & 57.1 & & & & & \\
\hline 330.0 & 40 & 332 & 9 & 2 & 50.0 & & & 328 & 47 & 333 \\
\hline 331.0 & 81 & 333 & 3 & 1 & 100.0 & & & 331.0 & 81 & 333 \\
\hline 338.0 & 53 & 353 & 7 & 2 & 50.0 & 24 & 1 & 338 & 53 & 353 \\
\hline 345.0 & 72 & 348 & 10 & 3 & 66.7 & \multirow{3}{*}{25} & \multirow{3}{*}{3} & 345.0 & 72 & 348 \\
\hline 346.0 & 75 & 257 & 3 & 1 & 100.0 & & & 346.0 & 75 & 257 \\
\hline 348.0 & 26 & 347 & 11 & 3 & 66.7 & & & 348.0 & 26 & 347 \\
\hline 355.0 & 30 & 348 & 16 & 5 & 60.0 & \multirow{4}{*}{26} & \multirow{4}{*}{2} & & & \\
\hline 356.0 & 28 & 349 & 8 & 2 & 100.0 & & & & & \\
\hline 358.0 & 30 & 355 & 7 & 2 & 100.0 & & & 356 & 29 & 351 \\
\hline 360.0 & 58 & 13 & 9 & 3 & 66.7 & & & 360.0 & 58 & 373 \\
\hline
\end{tabular}


1: Slope direction that was found to have one or more fracture planes dipping in a similar $\left( \pm 20^{\circ}\right)$ direction $($ column 1$)$

2: Dip angle for the fracture plane dipping in a direction similar to that of the slope

3: Dip direction for the fracture plane dipping in a direction similar to that of the slope

4: Total number of fracture planes that were measured at locations where one or more of the measured fracture planes dip in a direction similar to that of the slope

5: The number of locations where the fracture planes referred to in column 4 were measured.

6: The percentage of measured fracture planes that have dip directions similar to that of the slope direction

7: Group number, where each group refers to consecutive (three or more) slope directions that satisfied the condition of having one or more fracture planes dipping in a similar direction

8: Number of subgroups within each of the identified groups, where each subgroup includes fracture planes with similar dip directions

9: The average slope direction for each of the identified subgroups

10: The average fracture dip angle for each of the identified subgroups

11: The average fracture dip direction for each of the identified subgroups 